\title{
Smoothness Parameter of Power of Euclidean Norm
}

\author{
Anton Rodomanov ${ }^{1}$. Yurii Nesterov ${ }^{2}$
}

Received: 29 July 2019 / Accepted: 6 March 2020 / Published online: 27 March 2020

(c) The Author(s) 2020

\begin{abstract}
In this paper, we study derivatives of powers of Euclidean norm. We prove their Hölder continuity and establish explicit expressions for the corresponding constants. We show that these constants are optimal for odd derivatives and at most two times suboptimal for the even ones. In the particular case of integer powers, when the Hölder continuity transforms into the Lipschitz continuity, we improve this result and obtain the optimal constants.
\end{abstract}

Keywords Hölder continuity · Polynomials · Optimal constants

Mathematics Subject Classification 26A16 · 46G05 · 11C08

\section{Introduction}

Starting from the paper [1], there has been an increasing interest in the cubic regularization of Newton's method (see, for example, [2-8]), which has some attractive global worst-case complexity guarantees. The main idea of this method is to approximate the objective function with its second-order Taylor approximation, add to it the cube of Euclidean norm with certain coefficient and then minimize the result to obtain a new point.

A natural generalization of this approach consists in considering a general highorder Taylor approximation together with a certain high-order power of Euclidean norm as a regularizer. This leads to tensor methods [9-12] that have recently gained

Communicated by Liqun Qi.

$凶$ Anton Rodomanov

anton.rodomanov@uclouvain.be

Yurii Nesterov

yurii.nesterov@uclouvain.be

1 ICTEAM, Catholic University of Louvain, Louvain-la-Neuve, Belgium

2 Center for Operations Research and Economics, Catholic University of Louvain, Louvain-la-Neuve, Belgium 
their popularity after it was shown in [13] that one step of the third-order tensor method for minimizing convex functions is comparable with that of the cubic Newton method.

For some applications, involving functions with Hölder continuous derivatives, it may also be reasonable to regularize the models with fractional degrees of the Euclidean norm, as discussed in [14,15].

The efficiency of all the aforementioned methods strongly depends on our possibilities in solving the corresponding auxiliary problems that arise at each iteration. Therefore, it is important to be able to quickly solve minimization problems regularized by powers of Euclidean norm.

Two of the most important characteristics of the objective function that influence the convergence rate of minimization algorithms are the constants of uniform convexity and Hölder continuity of derivatives. It is thus important to know these parameters for powers of Euclidean norm in order to justify the convergence rates of the related minimization algorithms.

The uniform convexity of powers of Euclidean norm was first investigated in [16], where the authors obtained optimal constants for all integer powers. This result was then generalized to arbitrary real powers in [17, Lemma 5]. Thus, the question of uniform convexity is completely solved.

The question of the Hölder continuity of derivatives of powers of Euclidean norm is more subtle. There exist only partial results for some special powers. For example, for any real power between one and two, the Hölder continuity of the first derivative follows from the duality between uniform convexity and Hölder smoothness (see [18, Lemma 1]). For any real power between two and three, the Hölder continuity of the second derivative has recently been proved in [17, Example 2], where some suboptimal constants have been obtained. However, there are currently no general results for an arbitrary power.

Thus, establishing Hölder continuity of derivatives of powers of Euclidean norm and estimating the corresponding constants is still an open problem and constitutes the main topic of this work.

This paper is organized as follows. In Sect. 2, we introduce notation and recall important facts on the norm of symmetric multilinear operators.

In Sect. 3, we derive a general formula for derivatives of powers of Euclidean norm (Theorem 3.1). The main object in this formula is a certain family of recursively defined polynomials (Definition 3.1). We give the corresponding definition and provide several examples.

In Sects. 4 and 5, we study these polynomials in more detail. We establish useful identities and prove several important properties such as symmetry (Proposition 4.1), nonnegativity (Proposition 4.3) and monotonicity (Proposition 4.4). Section 5 is devoted to estimating the Hölder constants of the polynomials. The main results in this section are Theorems 5.1 and 5.2.

In Sect. 6, we apply the auxiliary results obtained in the previous sections for proving Hölder continuity of derivatives of powers of Euclidean norm. Namely, in Theorem 6.1, we derive a lower bound for the possible values of Hölder constants. In Theorem 6.2, we prove Hölder continuity of the derivatives along the lines passing through the origin. Finally, in Theorem 6.3, we extend this result onto the whole space and discuss the optimality of the constants. 
Finally, in Sect. 7, we show how to improve our general result for integer powers, when the Hölder condition corresponds to the Lipschitz condition.

\section{Notation and Generalities}

In this text, $\mathbb{E}$ is a finite-dimensional real vector space. Its dual space, composed of all linear functionals on $\mathbb{E}$, is denoted by $\mathbb{E}^{*}$. The value of a linear functional $s \in \mathbb{E}^{*}$, evaluated at a point $x \in \mathbb{E}$, is denoted by $\langle s, x\rangle$. To introduce a Euclidean norm $\|\cdot\|$ on $\mathbb{E}$, we fix a self-adjoint positive definite operator $B: \mathbb{E} \rightarrow \mathbb{E}^{*}$ and define $\|x\|:=\langle B x, x\rangle^{1 / 2}$.

For a function $f: G \rightarrow \mathbb{R}$, defined on an open set $G$ in $\mathbb{E}$, and for an integer $p \geq 0$, the $p$ th derivative of $f$, if exists, is denoted by $D^{p} f$. This derivative is a mapping from $G$ to the space of symmetric $p$-multilinear forms on $\mathbb{E}$.

Let $L$ be a $p$-multilinear form on $\mathbb{E}$. Its value, evaluated at $h_{1}, \ldots, h_{p} \in \mathbb{E}$, is denoted by $L\left[h_{1}, \ldots, h_{p}\right]$. When $h_{1}=\cdots=h_{p}=h$ for some $h \in \mathbb{E}$, we abbreviate this as $L[h]^{p}$. The norm of $L$ is defined in the standard way:

$$
\|L\|:=\max _{\left\|h_{1}\right\|=\cdots=\left\|h_{p}\right\|=1}\left|L\left[h_{1}, \ldots, h_{p}\right]\right| .
$$

If the form $L$ is symmetric, it is known that the maximum in the above definition can be achieved when all the vectors are the same:

$$
\|L\|=\max _{\|h\|=1}\left|L[h]^{p}\right|
$$

(see, for example, Appendix 1 in [19]).

For $q \in \mathbb{R}$, by $f_{q}: \mathbb{E} \rightarrow \mathbb{R}$ we denote the $q$ th power of the Euclidean norm:

$$
f_{q}(x):=\|x\|^{q} .
$$

The main goal of this paper is to establish that, for any integer $p \geq 0$ and any real $v \in[0,1]$, the $p$ th derivative of $f_{p+v}$ is $v$-Hölder continuous:

$$
\left\|D^{p} f_{p+v}\left(x_{2}\right)-D^{p} f_{p+v}\left(x_{1}\right)\right\| \leq A_{p, v}\left\|x_{2}-x_{1}\right\|
$$

for all $x_{1}, x_{2} \in \mathbb{E}$, where $A_{p, v}$ is an explicit constant dependent on $p$ and $\nu$.

\section{Derivatives of Powers of Euclidean Norm}

We start with deriving a general formula for derivatives of the function $f_{q}$. The main objects in this formula are univariate polynomials, defined below. 
Definition 3.1 For each integer $p \geq 0$ and each $q \in \mathbb{R}$, we define a polynomial $g_{p, q}: \mathbb{R} \rightarrow \mathbb{R}$ as follows. When $p=0$, we set $g_{p, q}(\tau):=1$. For all other $p \geq 1$,

$$
g_{p, q}(\tau):=\left(1-\tau^{2}\right) g_{p-1, q}^{\prime}(\tau)+(q-p+1) \tau g_{p-1, q}(\tau)
$$

Each polynomial $g_{p, q}$ is a combination of the previous polynomial $g_{p-1, q}$ and its derivative $g_{p-1, q}^{\prime}$. The first five polynomials can be written explicitly:

$$
\begin{aligned}
& g_{0, q}(\tau)=1, \quad g_{1, q}(\tau)=q \tau, \quad g_{2, q}(\tau)=q\left[(q-2) \tau^{2}+1\right], \\
& g_{3, q}(\tau)=q(q-2)\left[(q-4) \tau^{3}+3 \tau\right], \\
& g_{4, q}(\tau)=q(q-2)\left[(q-4)(q-6) \tau^{4}+6(q-4) \tau^{2}+3\right] .
\end{aligned}
$$

Let us now describe how derivatives of $f_{q}$ are related to polynomials $g_{p, q}$.

Theorem 3.1 For any real $q \in \mathbb{R}$, the function $f_{q}$ is $p$ times differentiable for all integer $0 \leq p<q$. The corresponding derivatives are

$$
D^{p} f_{q}(x)[h]^{p}=\|x\|^{q-p} g_{p, q}\left(\tau_{h}(x)\right),
$$

where $h \in \mathbb{E}$ is an arbitrary unit vector and

$$
\tau_{h}(x):= \begin{cases}\frac{\langle B x, h\rangle}{\|x\|}, & \text { if } x \neq 0 \\ 0, & \text { if } x=0 .\end{cases}
$$

Proof Note that $f_{q}$ is infinitely differentiable on $\mathbb{E} \backslash\{0\}$ since its restriction on this set is a composition of two infinitely differentiable functions, namely the quadratic function $\mathbb{E} \backslash\{0\} \rightarrow \mathbb{R}: x \mapsto\|x\|^{2}=\langle B x, x\rangle$ and the power function $] 0,+\infty\left[\rightarrow \mathbb{R}: t \mapsto t^{q / 2}\right.$. Hence, we only need to prove that $f_{q}$ is also $p$ times differentiable at the origin for any $0 \leq p<q$, and that (2) holds.

We proceed by induction. The case $p=0$ is trivial since, by definition, the zeroth derivative of a function is the function itself, while $g_{0, q}(\tau)=1$ for any $\tau \in \mathbb{R}$. Let us assume that $p \geq 1$, and the claim is proved for $p^{\prime}:=p-1$.

First, let us justify (2) for any $x \in \mathbb{E} \backslash\{0\}$. By the induction hypothesis,

$$
D^{p-1} f_{q}(x)[h]^{p-1}=\|x\|^{q-p+1} g_{p-1, q}\left(\tau_{h}(x)\right)
$$

for all $x \in \mathbb{E}$. On differentiating, we obtain that

$$
D\|\cdot\|(x)[h]=\tau_{h}(x), \quad D \tau_{h}(x)[h]=\frac{1-\tau_{h}^{2}(x)}{\|x\|}
$$


for all $x \in \mathbb{E} \backslash\{0\}$, and hence,

$$
\begin{aligned}
D^{p} f_{q}(x)[h]^{p}= & \|x\|^{q-p+1} g_{p-1, q}^{\prime}\left(\tau_{h}(x)\right) \frac{1-\tau_{h}^{2}(x)}{\|x\|} \\
& +(q-p+1)\|x\|^{q-p} \tau_{h}(x) g_{p-1, q}\left(\tau_{h}(x)\right) \\
= & \|x\|^{q-p}\left[\left(1-\tau_{h}^{2}(x)\right) g_{p-1, q}^{\prime}\left(\tau_{h}(x)\right)+\tau_{h}(x) g_{p-1, q}\left(\tau_{h}(x)\right)\right] \\
= & \|x\|^{q-p} g_{p, q}\left(\tau_{h}(x)\right),
\end{aligned}
$$

where the last equality follows from Definition 3.1.

Now let us show that $f_{q}$ is also $p$ times differentiable at the origin with $D^{p} f_{q}(0)=$ 0 . [This is what (2) says when $x=0$.] By our inductive assumption, we already know that $D^{p-1} f_{q}(0)=0$. Therefore, according to the definition of derivative, it remains to show that $\lim _{x \rightarrow 0 ; x \neq 0} \frac{\left\|D^{p-1} f_{q}(x)\right\|}{\|x\|}=0$, or, equivalently, in view of (1), that

$$
\lim _{x \rightarrow 0 ; x \neq 0} \max _{\|h\|=1} \frac{\left|D^{p-1} f_{q}(x)[h]^{p-1}\right|}{\|x\|}=0 .
$$

Applying our inductive assumption, we obtain that

$$
\max _{\|h\|=1} \frac{\left|D^{p-1} f_{q}(x)[h]^{p-1}\right|}{\|x\|}=\|x\|^{q-p} \max _{\|h\|=1}\left|g_{p-1, q}\left(\tau_{h}(x)\right)\right|
$$

for all $x \in \mathbb{E} \backslash\{0\}$. Since $p<q$, we have $\|x\|^{q-p} \rightarrow 0$ as $x \rightarrow 0$. Thus, we need to show that $\left|g_{p-1, q}\left(\tau_{h}(x)\right)\right|$ is uniformly bounded for all $x \in \mathbb{E}$ and all unit $h \in \mathbb{E}$. Indeed, by Cauchy-Schwartz inequality, we have $\left|\tau_{h}(x)\right| \leq 1$. Hence, $\left|g_{p-1, q}\left(\tau_{h}(x)\right)\right| \leq \max _{[-1,1]}\left|g_{p-1, q}\right|$. The right-hand side in the above inequality is finite, since a continuous function always achieves its maximum on a compact interval.

\section{Main Properties of Polynomials}

Let us study the polynomials $g_{p, q}$ introduced in Definition 3.1. Our first observation is that $g_{p, q}$, as a function, is always either even or odd.

Proposition 4.1 For any integer $p \geq 0$, and any $q \in \mathbb{R}, g_{p, q}$ has the same parity as p, i.e., $g_{p, q}(-\tau)=(-1)^{p} g_{p, q}(\tau)$ for all $\tau \in \mathbb{R}$.

Proof Easily follows from Definition 3.1 by induction.

Next we establish identities with the first and second derivatives of $g_{p, q}$.

Lemma 4.1 For any integer $p \geq 1$, and any $q, \tau \in \mathbb{R}$,

$$
g_{p, q}^{\prime}(\tau)=\left(1-\tau^{2}\right) g_{p-1, q}^{\prime \prime}(\tau)+(q-p-1) \tau g_{p-1, q}^{\prime}(\tau)+(q-p+1) g_{p-1, q}(\tau)
$$


Proof Follows from Definition 3.1 using standard rules of differentiation.

Lemma 4.2 For any integer $p \geq 0$, and any $q, \tau \in \mathbb{R}$,

$$
(q-p) g_{p, q}(\tau)=\tau g_{p, q}^{\prime}(\tau)+q g_{p, q-2}(\tau)
$$

Proof We proceed by induction on $p$. For $p=0$, by Definition 3.1, we have $(q-$ p) $g_{p, q}(\tau)=q$ while $\tau g_{p, q}^{\prime}(\tau)=0$ and $q g_{p, q-2}(\tau)=q$, so the claim is obviously true. Now let us prove the claim for $p \geq 1$, assuming that it is already true for all integer $0 \leq p^{\prime} \leq p-1$. By Definition 3.1, we have

$$
(q-p) g_{p, q}(\tau)=(q-p)\left(\left(1-\tau^{2}\right) g_{p-1, q}^{\prime}(\tau)+(q-p+1) \tau g_{p-1, q}(\tau)\right) .
$$

Rearranging, we obtain

$$
\begin{aligned}
(q-p) g_{p, q}(\tau)= & (q-p-1) \tau(q-p+1) g_{p-1, q}(\tau) \\
& +\left(1-\tau^{2}\right)(q-p) g_{p-1, q}^{\prime}(\tau)+(q-p+1) \tau g_{p-1, q}(\tau) .
\end{aligned}
$$

By the induction hypothesis, applied for $p^{\prime}:=p-1$, we have

$$
(q-p+1) g_{p-1, q}(\tau)=\tau g_{p-1, q}^{\prime}(\tau)+q g_{p-1, q-2}(\tau)
$$

for all $\tau \in \mathbb{R}$. Differentiating both sides, we obtain from this that

$$
(q-p) g_{p-1, q}^{\prime}(\tau)=\tau g_{p-1, q}^{\prime \prime}(\tau)+q g_{p-1, q-2}^{\prime}(\tau) .
$$

Combining the above three formulas, we see that

$$
\begin{aligned}
(q-p) g_{p, q}(\tau)= & (q-p-1) \tau\left(\tau g_{p-1, q}^{\prime}+q g_{p-1, q-2}(\tau)\right) \\
& +\left(1-\tau^{2}\right)\left(\tau g_{p-1, q}^{\prime \prime}(\tau)+q g_{p-1, q-2}^{\prime}(\tau)\right) \\
& +(q-p+1) \tau g_{p-1, q}(\tau) .
\end{aligned}
$$

At the same time, by Lemma 4.1, we have

$$
\tau g_{p, q}^{\prime}(\tau)=\left(1-\tau^{2}\right) \tau g_{p-1, q}^{\prime \prime}(\tau)+(q-p-1) \tau^{2} g_{p-1, q}^{\prime}(\tau)+(q-p+1) \tau g_{p-1, q}(\tau),
$$

and, by Definition 3.1, we also have

$$
q g_{p, q-2}(\tau)=\left(1-\tau^{2}\right) q g_{p-1, q-2}^{\prime}(\tau)+(q-p-1) \tau q g_{p-1, q-2}(\tau) .
$$

Summing the above two identities, we obtain the right-hand side of (6).

Lemma 4.3 For any integer $p \geq 1$, and any $q, \tau \in \mathbb{R}$,

$$
g_{p, q}^{\prime}(\tau)=\left(1-\tau^{2}\right) g_{p-1, q}^{\prime \prime}(\tau)+(q-p) \tau g_{p-1, q}^{\prime}(\tau)+q g_{p-1, q-2}(\tau) .
$$


Proof Apply Lemma 4.2 to the last term in (5).

The following lemma is particularly interesting. It turns out that, up to a constant factor, the derivative of the polynomial $g_{p, q}$ is exactly the previous polynomial but with a shifted value of $q$.

Lemma 4.4 For any integer $p \geq 1$, and any $q \in \mathbb{R}$, we have $g_{p, q}^{\prime}=p q g_{p-1, q-2}$.

Proof We proceed by induction on $p$. Let $\tau \in \mathbb{R}$. For $p=1$, we know from Definition 3.1 that $g_{p, q}(\tau)=q \tau$, while $p q g_{p-1, q-2}(\tau)=q$; therefore, the claim is indeed true. Now let us prove the claim for $p \geq 2$, assuming that it is already proved for all integer $0 \leq p^{\prime} \leq p-1$. From Lemma 4.3, we already know that

$$
g_{p, q}^{\prime}(\tau)=\left(1-\tau^{2}\right) g_{p-1, q}^{\prime \prime}(\tau)+(q-p) \tau g_{p-1, q}^{\prime}(\tau)+q g_{p-1, q-2}(\tau) .
$$

Therefore, it remains to prove that

$$
\left(1-\tau^{2}\right) g_{p-1, q}^{\prime \prime}(\tau)+(q-p) \tau g_{p-1, q}^{\prime}(\tau)=(p-1) q g_{p-1, q-2}(\tau) .
$$

By the induction hypothesis for $p^{\prime}:=p-1$, we already have the identity $g_{p-1, q}^{\prime}=$ $(p-1) q g_{p-2, q-2}$ and in particular $g_{p-1, q}^{\prime \prime}=(p-1) q g_{p-2, q-2}^{\prime}$. Thus,

$$
\begin{aligned}
& \left(1-\tau^{2}\right) g_{p-1, q}^{\prime \prime}(\tau)+(q-p) \tau g_{p-1, q}^{\prime}(\tau) \\
& \quad=(p-1) q\left[\left(1-\tau^{2}\right) g_{p-2, q-2}^{\prime}(\tau)+(q-p) \tau g_{p-2, q-2}(\tau)\right] .
\end{aligned}
$$

It remains to verify that

$$
\left(1-\tau^{2}\right) g_{p-2, q-2}^{\prime}(\tau)+(q-p) \tau g_{p-2, q-2}(\tau)=g_{p-1, q-2}(\tau)
$$

But this is given directly by Definition 3.1.

Combined with Definition 3.1, Lemma 4.4 gives us a useful recursive formula for $g_{p, q}$ that does not involve any derivatives.

Lemma 4.5 For any integer $p \geq 2$, and any $q, \tau \in \mathbb{R}$,

$$
g_{p, q}(\tau)=\left(1-\tau^{2}\right)(p-1) q g_{p-2, q-2}(\tau)+(q-p+1) \tau g_{p-1, q}(\tau) .
$$

Lemma 4.5 has several corollaries. The first one gives us closed-form expressions for the values of $g_{p, q}$ at the boundary points of the interval $[0,1]$.

Proposition 4.2 For any integer $p \geq 0$, and any $q \in \mathbb{R}$, we have 1

$$
g_{p, q}(0)= \begin{cases}(p-1) ! ! \prod_{i=0}^{\frac{p}{2}-1}(q-2 i), & \text { if } p \text { even } \\ 0, & \text { if } p \text { odd }\end{cases}
$$

\footnotetext{
1 For a positive integer $n$, by $n$ !! we denote the double factorial of $n$ (the product of all integers between 1 and $n$ with the same parity as $n)$. We also define $(-1) ! !=0 ! !=1$.
} 
and

$$
g_{p, q}(1)=\prod_{i=0}^{p-1}(q-i) .
$$

Proof We proceed by induction on $p$. From Definition 3.1, we have $g_{0, q}(0)=$ $g_{0, q}(1)=1$ and $g_{1, q}(0)=0, g_{1, q}(1)=q$. Thus, the claim is indeed true for $p=0$ and $p=1$. Now let us prove the claim for $p \geq 2$, assuming that it is already true for all integer $0 \leq p^{\prime} \leq p-1$. Using Lemma 4.5, we obtain

$$
g_{p, q}(0)=(p-1) q g_{p-2, q-2}(0)
$$

By the induction hypothesis, applied for $p^{\prime}:=p-2$ (and $q^{\prime}:=q-2$ ), we have

$$
g_{p-2, q-2}(0)= \begin{cases}(p-3) ! ! \prod_{i=0}^{\frac{p}{2}-2}(q-2-2 i), & \text { if } p \text { is even } \\ 0, & \text { if } p \text { is odd }\end{cases}
$$

By shifting the index in the product, this can be rewritten as

$$
g_{p-2, q-2}(0)= \begin{cases}(p-3) ! ! \prod_{i=1}^{\frac{p}{2}-1}(q-2 i), & \text { if } p \text { is even, } \\ 0, & \text { if } p \text { is odd }\end{cases}
$$

Substituting this into (10), we obtain (8).

Similarly, by Lemma 4.5, we also have $g_{p, q}(1)=(q-p+1) g_{p-1, q}(1)$. But by the induction hypothesis, $g_{p-1, q}(1)=\prod_{i=0}^{p-2}(q-i)$, and we obtain (9).

The second corollary of Lemma 4.5 states that $g_{p, q}$ cannot take negative values on the interval $[0,1]$, provided that $q$ is sufficiently large.

Proposition 4.3 For any integer $p \geq 0$, and any real $q \geq p-1, g_{p, q}$ is nonnegative on $[0,1]$.

Proof We proceed by induction on $p$. Let $0 \leq \tau \leq 1$. For $p=0$, we know, by Definition 3.1, that $g_{p, q}(\tau)=1$, which is actually nonnegative for all real $q$. For $p=1$, by Definition 3.1, we have $g_{p, q}(\tau)=q \tau$, which is indeed nonnegative when $q \geq p-1=0$.

Now let us prove the claim for $p \geq 2$, assuming that it is already proved for all integer $0 \leq p^{\prime} \leq p-1$. From Lemma 4.5, we know that

$$
g_{p, q}(\tau)=\left(1-\tau^{2}\right)(p-1) q g_{p-2, q-2}(\tau)+(q-p+1) \tau g_{p-1, q}(\tau) .
$$

By the induction hypothesis, applied, respectively, for $p^{\prime}:=p-2, q^{\prime}:=q-2$ and $p^{\prime}:=p-1, q^{\prime}:=q$ (observe that in both cases $q^{\prime} \geq p^{\prime}-1$ since $q \geq p$ ), we have $g_{p-2, q-2}(\tau) \geq 0$ and $g_{p-1, q}(\tau) \geq 0$. Since $q \geq p-1 \geq 1$, then also $q-p+1 \geq 0$, and $(p-1) q \geq 0$. Thus, all parts in the right-hand side of the above formula are nonnegative. 
Combining Proposition 4.3 with Lemma 4.4, we obtain that, when $q \geq p$, the polynomial $g_{p, q}$ is not only nonnegative but also monotonically increasing.

Proposition 4.4 For any integer $p \geq 0$, and any real $q \geq p$, the derivative $g_{p, q}^{\prime}$ is nonnegative on $[0,1]$; hence $g_{p, q}$ is monotonically increasing on $[0,1]$.

Finally, let us show how we can apply the properties that we have established above, to find the maximal absolute value of $g_{p, q}$ on $[-1,1]$.

Proposition 4.5 For any integer $p \geq 0$, and any real $q \geq p$,

$$
\max _{[-1,1]}\left|g_{p, q}\right|=\prod_{i=0}^{p-1}(q-i) .
$$

Proof By Proposition 4.1, we have $\max _{[-1,1]}\left|g_{p, q}\right|=\max _{[0,1]}\left|g_{p, q}\right|$. Since $g_{p, q}$ is nonnegative on $[0,1]$ (Proposition 4.3), $\max _{[0,1]}\left|g_{p, q}\right|=\max _{[0,1]} g_{p, q}$. By Proposition 4.4, $\max _{[0,1]} g_{p, q}=g_{p, q}(1)$. But $g_{p, q}(1)=\prod_{i=0}^{p-1}(q-i)$ according to Proposition 4.2.

\section{Hölder Constants of Polynomials}

We continue our study of polynomials $g_{p, q}$, but now we restrict our attention to the particular case when $q=p+v$ for some real $v \in[0,1]$.

Clearly, the polynomial $g_{p, p+v}$ is $v$-Hölder continuous on $[-1,1]$, since this is true for any other polynomial on a compact interval. The goal of this section is to obtain an explicit expression for the corresponding Hölder constant. We start with the result, allowing us to reduce our task to that on $[0,1]$.

Theorem 5.1 For any integer $p \geq 0$, and any real $v \in[0,1]$, the polynomial $g_{p, p+v}$ is $v$-Hölder continuous on $[-1,1]$ with constant

$$
\tilde{H}_{p, v}:= \begin{cases}H_{p, v}, & \text { if } p \text { is even } \\ 2^{1-v} H_{p, v}, & \text { if } p \text { is odd }\end{cases}
$$

where $H_{p, v}$ is the corresponding Hölder constant of $g_{p, p+v}$ on $[0,1]$.

Proof Let $\tau_{1}, \tau_{2} \in[-1,1]$. We need to prove that

$$
\left|g_{p, p+v}\left(\tau_{2}\right)-g_{p, p+v}\left(\tau_{1}\right)\right| \leq \tilde{H}_{p, v}\left|\tau_{2}-\tau_{1}\right|^{v}
$$

By Proposition 4.1, this inequality is invariant to negation transformations $\left(\tau_{1}, \tau_{2}\right) \mapsto\left(-\tau_{1},-\tau_{2}\right)$. Therefore, we can assume that $\tau_{2} \geq 0$. Furthermore, we can assume that $\tau_{1}<0$, since otherwise the claim is trivial.

Case I Suppose $p$ is even. Then, by Proposition 4.1,

$$
\left|g_{p, p+v}\left(\tau_{2}\right)-g_{p, p+v}\left(\tau_{1}\right)\right|=\left|g_{p, p+v}\left(\tau_{2}\right)-g_{p, p+v}\left(-\tau_{1}\right)\right|
$$


Note that $-\tau_{1}, \tau_{2} \in[0,1]$. Therefore, by Hölder condition on $[0,1]$,

$$
\left|g_{p, p+v}\left(\tau_{2}\right)-g_{p, p+v}\left(-\tau_{1}\right)\right| \leq H_{p, v}\left|\tau_{2}+\tau_{1}\right|^{v} .
$$

At the same time, $\left|\tau_{2}+\tau_{1}\right| \leq \tau_{2}-\tau_{1}$ by the triangle inequality, and (11) follows.

Case II Now suppose $p$ is odd. By Propositions 4.1 and 4.3,

$$
\left|g_{p, p+v}\left(\tau_{2}\right)-g_{p, p+v}\left(\tau_{1}\right)\right|=g_{p, p+v}\left(\tau_{2}\right)+g_{p, p+v}\left(-\tau_{1}\right)
$$

Recall that $g_{p, p+v}(0)=0$ (Proposition 4.2). Therefore,

$$
\begin{aligned}
g_{p, p+v}\left(\tau_{2}\right) & =g_{p, p+v}\left(\tau_{2}\right)-g_{p, p+v}(0) \leq H_{p, v} \tau_{2}^{v}, \\
g_{p, p+v}\left(-\tau_{1}\right) & =g_{p, p+v}\left(-\tau_{1}\right)-g_{p, p+v}(0) \leq H_{p, v}\left(-\tau_{1}\right)^{v} .
\end{aligned}
$$

Hence,

$$
g_{p, p+v}\left(\tau_{2}\right)+g_{p, p+v}\left(-\tau_{1}\right) \leq H_{p, v}\left(\tau_{2}^{v}+\left(-\tau_{1}\right)^{v}\right)
$$

To prove (11), it remains to show that $\tau_{2}^{v}+\left(-\tau_{1}\right)^{v} \leq 2^{1-v}\left(\tau_{2}-\tau_{1}\right)^{v}$. But this follows from the concavity of power function $t \mapsto t^{\nu}$.

Our next task is to estimate the Hölder constant of $g_{p, p+v}$ on $[0,1]$ :

$$
H_{p, v}:=\max _{0 \leq \tau_{1}<\tau_{2} \leq 1} \frac{g_{p, p+v}\left(\tau_{2}\right)-g_{p, p+v}\left(\tau_{1}\right)}{\left(\tau_{2}-\tau_{1}\right)^{v}} .
$$

Note that Proposition 4.4 allows us to remove the absolute value sign.

Theorem 5.2 For any integer $p \geq 0$, and any real $v \in[0,1]$, we have

$$
H_{p, v} \leq \prod_{i=1}^{p}(v+i)
$$

The proof of Theorem 5.2 is based on two auxiliary propositions.

Proposition 5.1 For any integer $p \geq 0$ and any real $\nu, \tau_{1} \in[0,1]$, the function

$$
] \tau_{1},+\infty\left[\rightarrow \mathbb{R}: \tau_{2} \mapsto \frac{g_{p, p+v}\left(\tau_{2}\right)-g_{p, p+v}\left(\tau_{1}\right)}{\left(\tau_{2}-\tau_{1}\right)^{v}}\right.
$$

is monotonically increasing on $\left.] \tau_{1}, 1\right]$.

Proposition 5.2 For any integer $p \geq 0$ and any real $v \in[0,1]$, the function

$$
] 0,1] \rightarrow \mathbb{R}: \tau \mapsto \frac{g_{p, p+v}(\tau)}{1-(1-\tau)^{v}}
$$

is monotonically decreasing on ]0, 1]. 
Let us assume for a moment that these propositions are already proved. Then, the proof of Theorem 5.2 is simple.

Proof Let $0 \leq \tau_{1}<\tau_{2} \leq 1$. From Proposition 5.1, we know that

$$
\frac{g_{p, p+v}\left(\tau_{2}\right)-g_{p, p+v}\left(\tau_{1}\right)}{\left(\tau_{2}-\tau_{1}\right)^{v}} \leq \frac{g_{p, p+v}(1)-g_{p, p+v}\left(\tau_{1}\right)}{\left(1-\tau_{1}\right)^{v}}
$$

Therefore, to prove (13), it remains to show that

$$
\frac{g_{p, p+v}(1)-g_{p, p+v}\left(\tau_{1}\right)}{\left(1-\tau_{1}\right)^{v}} \leq \prod_{i=1}^{p}(v+i)
$$

Recall that, by Proposition 4.2, we have $\prod_{i=1}^{p}(v+i)=g_{p, p+v}(1)$. Thus, the inequality we need to prove is

$$
\frac{g_{p, p+v}(1)-g_{p, p+v}\left(\tau_{1}\right)}{\left(1-\tau_{1}\right)^{v}} \leq g_{p, p+v}(1),
$$

or, equivalently,

$$
\frac{g_{p, p+v}\left(\tau_{1}\right)}{1-\left(1-\tau_{1}\right)^{v}} \geq g_{p, p+v}(1)
$$

But this follows from Proposition 5.2.

Our goal now is to prove Propositions 5.1 and 5.2.

We start with Proposition 5.1. It requires three technical lemmas.

Lemma 5.1 For any integer $p \geq 0$, and any real $\nu, \tau \in[0,1]$,

$$
g_{p, p+v}(\tau) \geq \tau g_{p, p+v}^{\prime}(\tau) .
$$

Moreover, when $p \geq 2$,

$$
\begin{aligned}
& g_{p, p+v}(\tau)-\tau g_{p, p+v}^{\prime}(\tau) \\
& \quad \geq\left(1-\tau^{2}\right)(p-1)(p+v)\left(g_{p-2, p-2+v}(\tau)-\tau g_{p-2, p-2+v}^{\prime}(\tau)\right) .
\end{aligned}
$$

Proof First, let us prove (17). By Lemma 4.1, we have

$$
g_{p, p+v}^{\prime}(\tau)=\left(1-\tau^{2}\right) g_{p-1, p+v}^{\prime \prime}(\tau)+(v-1) \tau g_{p-1, p+v}^{\prime}(\tau)+(v+1) g_{p-1, p+v}(\tau) .
$$

Since $g_{p-1, p+v}^{\prime}(\tau) \geq 0$ (Proposition 4.4) and $v \leq 1$, it follows that

$$
g_{p, p+v}^{\prime}(\tau) \leq\left(1-\tau^{2}\right) g_{p-1, p+v}^{\prime \prime}(\tau)+(v+1) g_{p-1, p+v}(\tau) .
$$


At the same time, by Definition 3.1,

$$
g_{p, p+v}(\tau)=\left(1-\tau^{2}\right) g_{p-1, p+v}^{\prime}(\tau)+(\nu+1) \tau g_{p-1, p+v}(\tau)
$$

Thus,

$$
g_{p, p+v}(\tau)-\tau g_{p, p+v}^{\prime}(\tau) \geq\left(1-\tau^{2}\right)\left(g_{p-1, p+v}^{\prime}(\tau)-\tau g_{p-1, p+v}^{\prime \prime}(\tau)\right) .
$$

Applying Lemma 4.4, we obtain that

$$
\begin{aligned}
& g_{p-1, p+v}^{\prime}(\tau)=(p-1)(p+v) g_{p-2, p-2+v}(\tau), \\
& g_{p-1, p+v}^{\prime \prime}(\tau)=(p-1)(p+v) g_{p-2, p-2+v}^{\prime}(\tau),
\end{aligned}
$$

and (17) follows.

It remains to prove (16). For $p=0$, we have $g_{p, p+v}(\tau)=1$ (Definition 3.1), and hence, $\tau g_{p, p+v}^{\prime}(\tau)=0$, and (16) is indeed true. For $p=1$, by Definition 3.1, we have $g_{p, p+v}(\tau)=(p+v) \tau$, and hence, $\tau g_{p, p+v}^{\prime}(\tau)=(p+v) \tau$, and (16) is again true. The general case $p \geq 2$ easily follows from (17) by induction.

Lemma 5.2 For any integer $p \geq 0$, any real $v \in[0,1]$, and $0 \leq \tau_{1} \leq \tau_{2} \leq 1$,

$$
(p+v) g_{p, p-2+v}\left(\tau_{2}\right) \leq v\left(g_{p, p+v}\left(\tau_{1}\right)-\tau_{1} g_{p, p+v}^{\prime}\left(\tau_{1}\right)\right)
$$

Proof We use induction in $p$. For $p=0$, we have $g_{p, p-2+v}\left(\tau_{2}\right)=1$, while $g_{p, p+v}\left(\tau_{1}\right)-\tau_{1} g_{p, p+v}^{\prime}\left(\tau_{1}\right)=1$ (see Definition 3.1), so the claim is true. For $p=1$, we have $g_{p, p-2+v}\left(\tau_{2}\right)=-(1-v) \tau_{2} \leq 0$ while $g_{p, p+v}\left(\tau_{1}\right)-\tau_{1} g_{p, p+v}^{\prime}\left(\tau_{1}\right)=0$, (see Definition 3.1), and hence, the claim is again true.

Now we prove the claim for $p \geq 2$, assuming that it is already true for all integer $0 \leq p^{\prime} \leq p-1$. According to Lemma 4.5, we have

$$
\begin{aligned}
g_{p, p-2+v}\left(\tau_{2}\right)= & \left(1-\tau_{2}^{2}\right)(p-1)(p-2+v) g_{p-2, p-4+v}\left(\tau_{2}\right) \\
& -(1-v) \tau_{2} g_{p-1, p-2+v}\left(\tau_{2}\right)
\end{aligned}
$$

Since $g_{p-1, p-2+v}\left(\tau_{2}\right) \geq 0$ (Proposition 4.3), we further have

$$
g_{p, p-2+v}\left(\tau_{2}\right) \leq\left(1-\tau_{2}^{2}\right)(p-1)(p-2+v) g_{p-2, p-4+v}\left(\tau_{2}\right) .
$$

If $g_{p-2, p-4+v}\left(\tau_{2}\right) \leq 0$, it follows that $g_{p, p-2+v}\left(\tau_{2}\right) \leq 0$, and the proof in this case is finished, because the right-hand side in (18) is always nonnegative in view of Lemma 5.1. Therefore, we can assume that $g_{p-2, p-4+v}\left(\tau_{2}\right) \geq 0$.

Since $\tau_{2} \geq \tau_{1}$, then

$$
g_{p, p-2+v}\left(\tau_{2}\right) \leq\left(1-\tau_{1}^{2}\right)(p-1)(p-2+v) g_{p-2, p-4+v}\left(\tau_{2}\right)
$$


Applying the inductive assumption to $p^{\prime}:=p-2$, we obtain

$$
(p-2+v) g_{p-2, p-4+v}\left(\tau_{2}\right) \leq v\left(g_{p-2, p-2+v}\left(\tau_{1}\right)-\tau_{1} g_{p-2, p-2+v}^{\prime}\left(\tau_{1}\right)\right) .
$$

Hence,

$$
g_{p, p-2+v}\left(\tau_{2}\right) \leq v\left(1-\tau_{1}^{2}\right)(p-1)\left(g_{p-2, p-2+v}\left(\tau_{1}\right)-\tau_{1} g_{p-2, p-2+v}^{\prime}\left(\tau_{1}\right)\right) .
$$

Thus, to finish the proof, it remains to show that

$$
\begin{aligned}
& \left(1-\tau_{1}^{2}\right)(p-1)(p+v)\left(g_{p-2, p-2+v}\left(\tau_{1}\right)-\tau_{1} g_{p-2, p-2+v}^{\prime}\left(\tau_{1}\right)\right) \\
& \quad \leq g_{p, p+v}\left(\tau_{1}\right)-\tau_{1} g_{p, p+v}^{\prime}\left(\tau_{1}\right) .
\end{aligned}
$$

But this is guaranteed by Lemma 5.1 .

Lemma 5.3 For any integer $p \geq 0$, and any real $v, \tau_{2} \in[0,1]$, the function

$$
] 0,+\infty\left[\rightarrow \mathbb{R}: \tau_{1} \mapsto \frac{\nu g_{p, p+v}\left(\tau_{1}\right)-(p+v) g_{p, p-2+v}\left(\tau_{2}\right)}{\tau_{1}}\right.
$$

is monotonically decreasing on $\left.] 0, \tau_{2}\right]$.

Proof The function (19) is differentiable with derivative

$$
\frac{v\left(\tau_{1} g_{p, p+v}^{\prime}\left(\tau_{1}\right)-g_{p, p+v}\left(\tau_{1}\right)\right)+(p+v) g_{p, p-2+v}\left(\tau_{2}\right)}{\tau_{1}^{2}},
$$

which is non-positive on $] 0, \tau_{2}$ ] by Lemma 5.2.

Now we can present the proof of Proposition 5.1:

Proof Since (14) is differentiable, it suffices to prove that its derivative

$$
\frac{g_{p, p+v}^{\prime}\left(\tau_{2}\right)}{\left(\tau_{2}-\tau_{1}\right)^{v}}-\frac{v\left(g_{p, p+v}\left(\tau_{2}\right)-g_{p, p+v}\left(\tau_{1}\right)\right)}{\left(\tau_{2}-\tau_{1}\right)^{v+1}}
$$

is nonnegative for all $0<\tau_{1}<\tau_{2} \leq 1$, or, equivalently, that

$$
g_{p, p+v}^{\prime}\left(\tau_{2}\right)\left(\tau_{2}-\tau_{1}\right) \geq v\left(g_{p, p+v}\left(\tau_{2}\right)-g_{p, p+v}\left(\tau_{1}\right)\right) .
$$

By Lemma 4.2,

$$
v g_{p, p+v}\left(\tau_{2}\right)=\tau_{2} g_{p, p+v}^{\prime}\left(\tau_{2}\right)+(p+v) g_{p, p-2+v}\left(\tau_{2}\right) .
$$

Therefore, it is enough to prove that

$$
v g_{p, p+v}\left(\tau_{1}\right)-(p+v) g_{p, p-2+v}\left(\tau_{2}\right) \geq \tau_{1} g_{p, p+v}^{\prime}\left(\tau_{2}\right),
$$


or, equivalently,

$$
\frac{v g_{p, p+v}\left(\tau_{1}\right)-(p+v) g_{p, p-2+v}\left(\tau_{2}\right)}{\tau_{1}} \geq g_{p, p+v}^{\prime}\left(\tau_{2}\right) .
$$

But this immediately follows from Lemma 5.3 using (20).

It remains to prove Proposition 5.2. For this, we need one more lemma.

Lemma 5.4 For any integer $p \geq 0$, and any real $v, \tau \in[0,1]$, we have

$$
(p+v) g_{p, p-2+v}(\tau) \geq-\left(1-(1-\tau)^{1-v}\right) g_{p, p+v}^{\prime}(\tau) .
$$

Proof As usual, we use induction in $p$. The base case $p=0$ is trivial, since $g_{p, p-2+v}(\tau)=1$, while $g_{p, p+v}^{\prime}(\tau)=0$ (see Definition 3.1). To prove the general case $p \geq 1$, we assume that (22) is already true for all integer $0 \leq p^{\prime} \leq p-1$.

Our first step is to show that

$$
\begin{aligned}
(p+v) g_{p, p-2+v}(\tau) \geq & -\left(1-\tau^{2}\right)\left(1-(1-\tau)^{1-\nu}\right) g_{p-1, p+v}^{\prime \prime}(\tau) \\
& -(p+v)(1-v) \tau g_{p-1, p-2+v}(\tau) .
\end{aligned}
$$

If $p=1$, we have $g_{p, p-2+v}(\tau)=-(1-v) \tau$, while $g_{p-1, p+v}^{\prime \prime}(\tau)=0$ and $g_{p-1, p-2+v}(\tau)=1$ (see Definition 3.1), so (23) is indeed true. To justify it for all other $p \geq 2$, we proceed as follows. By Lemma 4.5, we know that

$$
\begin{aligned}
g_{p, p-2+\nu}(\tau)= & \left(1-\tau^{2}\right)(p-1)(p-2+\nu) g_{p-2, p-4+v}(\tau) \\
& -(1-v) \tau g_{p-1, p-2+v}(\tau) .
\end{aligned}
$$

Therefore, (23) is equivalent to

$$
(p+v)(p-1)(p-2+v) g_{p-2, p-4+v}(\tau) \geq-\left(1-(1-\tau)^{1-v}\right) g_{p-1, p+v}^{\prime \prime}(\tau) .
$$

By our inductive assumption (22), applied to $p^{\prime}:=p-2$, we already have

$$
(p-2+v) g_{p-2, p-4+v}(\tau) \geq-\left(1-(1-\tau)^{1-v}\right) g_{p-2, p-2+v}^{\prime}(\tau) .
$$

At the same time, by Lemma 4.4,

$$
(p+v)(p-1) g_{p-2, p-2+v}^{\prime}(\tau)=g_{p-1, p+v}^{\prime \prime}(\tau) .
$$

Thus, (23) is established.

Now we estimate the right-hand side in (23). Applying Lemma 4.3 and the fact that $g_{p-1, p+v}^{\prime}(\tau) \geq 0$ (Proposition 4.4), we obtain

$$
\begin{aligned}
g_{p, p+v}^{\prime}(\tau) & =\left(1-\tau^{2}\right) g_{p-1, p+v}^{\prime \prime}(\tau)+v \tau g_{p-1, p+v}^{\prime}(\tau)+(p+v) g_{p-1, p-2+v}(\tau) \\
& \geq\left(1-\tau^{2}\right) g_{p-1, p+v}^{\prime \prime}(\tau)+(p+v) g_{p-1, p-2+v}(\tau)
\end{aligned}
$$


From this, it follows that

$$
\left(1-\tau^{2}\right) g_{p-1, p+v}^{\prime \prime}(\tau) \leq g_{p, p+v}^{\prime}(\tau)-(p+v) g_{p-1, p-2+v}(\tau) .
$$

Substituting the above equation into (23), we obtain

$$
\begin{aligned}
(p+v) g_{p, p-2+v}(\tau) \geq & -\left(1-(1-\tau)^{1-v}\right) g_{p, p+v}^{\prime}(\tau) \\
& +(p+v)\left(1-(1-v) \tau-(1-\tau)^{1-v}\right) g_{p-1, p-2+v}(\tau) .
\end{aligned}
$$

Since $g_{p-1, p-2+v}(\tau) \geq 0$ (by Proposition 4.3), it only remains to show that (1 $\tau)^{1-v} \leq 1-(1-v) \tau$. But this follows from the concavity of power function $\tau \mapsto$ $(1-\tau)^{1-v}$.

Now we can give the proof of Proposition 5.2:

Proof Since (15) is differentiable, it suffices to prove that its derivative

$$
\frac{\left(1-(1-\tau)^{v}\right) g_{p, p+v}^{\prime}(\tau)-v(1-\tau)^{\nu-1} g_{p, p+v}(\tau)}{\left(1-(1-\tau)^{v}\right)^{2}}
$$

is non-positive for all $0<\tau<1$. By Lemma 4.2, we have

$$
v g_{p, p+v}(\tau)=\tau g_{p, p+v}^{\prime}(\tau)+(p+v) g_{p, p-2+v}(\tau) .
$$

Thus, we need to show that

$$
(1-\tau)^{\nu-1}\left(\tau g_{p, p+v}^{\prime}(\tau)+(p+v) g_{p, p-2+v}(\tau)\right) \geq\left(1-(1-\tau)^{v}\right) g_{p, p+v}^{\prime}(\tau)
$$

or, equivalently (by multiplying both sides by $\left.(1-\tau)^{1-v}\right)$, that

$$
\tau g_{p, p+v}^{\prime}(\tau)+(p+v) g_{p, p-2+v}(\tau) \geq\left((1-\tau)^{1-v}-1+\tau\right) g_{p, p+v}^{\prime}(\tau),
$$

or, equivalently (by moving the first term into the right-hand side), that

$$
(p+v) g_{p, p-2+v}(\tau) \geq-\left(1-(1-\tau)^{1-v}\right) g_{p, p+v}^{\prime}(\tau) .
$$

But this is given by Lemma 5.4 .

To conclude this section, let us discuss the optimality of Theorem 5.2.

For odd values of $p$, the obtained constant $\prod_{i=1}^{p}(v+i)$ turns out to be optimal. Indeed, using $\tau_{1}:=0, \tau_{2}:=1$ in (12) and taking into account Proposition 4.2, we obtain that

$$
H_{p, v} \geq \frac{g_{p, p+v}\left(\tau_{2}\right)-g_{p, p+v}\left(\tau_{1}\right)}{\left(\tau_{2}-\tau_{1}\right)^{v}}=g_{p, p+v}(1)=\prod_{i=1}^{p}(v+i) .
$$


However, for even $p$, this constant is suboptimal. For example, consider the case when $p=2$. We know that

$$
g_{2,2+v}(\tau)=(v+2)\left(v \tau^{2}+1\right)
$$

The corresponding optimal constant, according to Proposition 5.1, is

$$
H_{2, v}=\max _{0 \leq \tau<1} \frac{g_{2,2+\nu}(1)-g_{2,2+\nu}(\tau)}{(1-\tau)^{v}}=v(\nu+2) \max _{0 \leq \tau<1}(1-\tau)^{1-v}(1+\tau) \text {. }
$$

Note that this maximization problem is logarithmically concave in $\tau$. Taking the logarithm and setting the derivative to zero, we find that the maximal point corresponds to $\tau:=\frac{v}{2-v} \in[0,1]$, and the corresponding optimal value is

$$
H_{2, v}=v(v+2) \frac{2^{2-v}(1-v)^{1-v}}{(2-v)^{2-v}} \leq(v+1)(v+2) \text {. }
$$

Of course, the last inequality is strict for all $0 \leq v<1$.

\section{Hölder Continuity of Derivatives of Powers of Euclidean Norm}

We have established the main properties of polynomials $g_{p, q}$ and obtained an explicit upper bound on their Hölder constant. Hence, we are ready to prove the Hölder continuity of derivatives of powers of Euclidean norm. Let us start with a simple result that gives us a lower bound on the Hölder constant.

Theorem 6.1 For any integer $p \geq 0$, and any real $v \in[0,1]$, the Hölder constant of $D^{p} f_{p+v}$, corresponding to degree $v$, cannot be smaller than

$$
C_{p, v}:= \begin{cases}\prod_{i=1}^{p}(v+i), & \text { if } p \text { is even } \\ 2^{1-v} \prod_{i=1}^{p}(v+i), & \text { if } p \text { is odd }\end{cases}
$$

Proof According to (1), we need to show that

$$
\left|D^{p} f_{p+v}\left(x_{2}\right)[h]^{p}-D^{p} f_{p+v}\left(x_{1}\right)[h]^{p}\right| \geq C_{p, v}\left\|x_{2}-x_{1}\right\|^{v}
$$

for some $x_{1}, x_{2} \in \mathbb{E}$ and some unit $h \in \mathbb{E}$. Let us choose an arbitrary unit vector $h \in \mathbb{E}$, and set $x_{2}:=h$. By Theorem 3.1 and Proposition 4.2,

$$
D^{p} f_{p+v}\left(x_{2}\right)[h]^{p}=\left\|x_{2}\right\|^{v} g_{p, p+v}(1)=\prod_{i=1}^{p}(v+i) .
$$

To specify $x_{1}$, we consider two cases. 
If $p$ is even, set $x_{1}:=0$. Then, $D^{p} f_{p+v}\left(x_{1}\right)[h]^{p}=0$ by Theorem 3.1 , and

$$
\left|D^{p} f_{p+v}\left(x_{2}\right)[h]^{p}-D^{p} f_{p+v}\left(x_{1}\right)[h]^{p}\right|=\prod_{i=1}^{p}(v+i),
$$

which is exactly $C_{p, v}\left\|x_{2}-x_{1}\right\|^{\nu}$. If $p$ is odd, we take $x_{1}:=-h$. This gives us

$$
D^{p} f_{p+v}\left(x_{1}\right)[h]^{p}=\left\|x_{1}\right\|^{v} g_{p, p+v}(-1)=-\prod_{i=1}^{p}(v+i),
$$

where we apply Proposition 4.1 to rewrite $g_{p, p+v}(-1)=g_{p, p+v}(1)$. Hence,

$$
\left|D^{p} f_{p+v}\left(x_{2}\right)[h]^{p}-D^{p} f_{p+v}\left(x_{1}\right)[h]^{p}\right|=2 \prod_{i=1}^{p}(v+i),
$$

which is again precisely $C_{p, v}\left\|x_{2}-x_{1}\right\|^{v}$.

Next we prove Hölder continuity with the optimal constant along any line, passing through the origin.

Theorem 6.2 For any integer $p \geq 0$, and any real $v \in[0,1]$, the restriction of $D^{p} f_{p+v}$ to a line, passing through the origin, is $v$-Hölder continuous with constant $C_{p, v}$.

Proof Let $x_{1}, x_{2} \in \mathbb{E}$ be arbitrary points, lying on a line, passing through the origin, and let $h \in \mathbb{E}$ be an arbitrary unit vector. According to (1) and Theorem 3.1, we need to show that

$$
\left|\left\|x_{2}\right\|^{v} g_{p, p+v}\left(\tau_{2}\right)-\left\|x_{1}\right\|^{v} g_{p, p+v}\left(\tau_{1}\right)\right| \leq C_{p, v}\left\|x_{2}-x_{1}\right\|^{v},
$$

where $\tau_{1}:=\tau_{h}\left(x_{1}\right), \tau_{2}:=\tau_{h}\left(x_{2}\right)$.

Observe that this inequality is symmetric in $x_{1}$ and $x_{2}$ and is invariant when we replace the pair $\left(x_{1}, x_{2}\right)$ with $\left(-x_{1},-x_{2}\right)$. Therefore, we can assume that $\left\|x_{2}\right\| \geq\left\|x_{1}\right\|$ and $\tau_{2} \geq 0$.

Since $x_{1}$ and $x_{2}$ lie on a line, passing through the origin, $\tau_{1}$ and $\tau_{2}$ can differ only in sign. Hence, by Proposition 4.1, we have two options: either $g_{p, p+v}\left(\tau_{1}\right)=g_{p, p+v}\left(\tau_{2}\right)$ or $g_{p, p+v}\left(\tau_{1}\right)=-g_{p, p+v}\left(\tau_{2}\right)$.

Case I Suppose $g_{p, p+v}\left(\tau_{1}\right)=g_{p, p+v}\left(\tau_{2}\right)$ (while $\tau_{1}$ can be of any sign). Then,

$$
\left|\left\|x_{2}\right\|^{v} g_{p, p+v}\left(\tau_{2}\right)-\left\|x_{1}\right\|^{v} g_{p, p+v}\left(\tau_{1}\right)\right|=\left|g_{p, p+v}\left(\tau_{2}\right)\right|\left(\left\|x_{2}\right\|^{v}-\left\|x_{1}\right\|^{v}\right) \text {. }
$$

By Proposition 4.5 and (24), we know that

$$
\left|g_{p, p+v}\left(\tau_{2}\right)\right| \leq \prod_{i=1}^{p}(v+i) \leq C_{p, v}
$$


Thus, it suffices to prove that $\left\|x_{2}\right\|^{v}-\left\|x_{1}\right\|^{v} \leq\left\|x_{2}-x_{1}\right\|^{v}$. But this follows from the well-known inequality $r_{2}^{v}-r_{1}^{v} \leq\left(r_{2}-r_{1}\right)^{v}$ (which is valid for any real $0 \leq r_{1} \leq r_{2}$ ) combined with the reverse triangle inequality.

Case II Suppose $g_{p, p+v}\left(\tau_{1}\right)=-g_{p, p+v}\left(\tau_{2}\right)(\neq 0)$. By Proposition 4.1 and Proposition 4.3 , this happens only if $p$ is odd and $\tau_{1} \leq 0$. Thus, $\tau_{1}=-\tau_{2}$, and

$$
\left|\left\|x_{2}\right\|^{v} g_{p, p+v}\left(\tau_{2}\right)-\left\|x_{1}\right\|^{v} g_{p, p+v}\left(\tau_{1}\right)\right|=\left|g_{p, p+v}\left(\tau_{2}\right)\right|\left(\left\|x_{2}\right\|^{v}+\left\|x_{1}\right\|^{v}\right) .
$$

Due to (25), it remains to prove that $\left\|x_{2}\right\|^{v}+\left\|x_{1}\right\|^{v} \leq 2^{1-v}\left\|x_{2}-x_{1}\right\|^{v}$. But this is immediate. Indeed, $\left\|x_{2}\right\|^{v}+\left\|x_{1}\right\|^{v} \leq 2^{1-v}\left(\left\|x_{2}\right\|+\left\|x_{1}\right\|\right)^{v}$ by the concavity of the power function $t \mapsto t^{\nu}$, while $\left\|x_{2}\right\|+\left\|x_{1}\right\|=\left\|x_{2}-x_{1}\right\|$ since the segment $\left[x_{1}, x_{2}\right]$ contains the origin..

Our final step is to extend Hölder continuity from lines, passing through the origin, onto the whole space. The main instrument for doing this is exploiting Hölder continuity of $g_{p, p+v}$ that we studied in Sect. 5 .

Theorem 6.3 For any integer $p \geq 0$, and any real $v \in[0,1], D^{p} f_{p+v}$ is $v$-Hölder continuous with constant

$$
A_{p, v}:= \begin{cases}(p-1) ! ! \prod_{i=1}^{p / 2}(v+2 i)+H_{p, v}, & \text { if } p \text { is even } \\ 2^{1-v} \prod_{i=1}^{p}(v+i), & \text { if } p \text { is odd }\end{cases}
$$

where $H_{p, v}$ is the constant of $v$-Hölder continuity of $g_{p, p+v}$ on $[0,1]$. In particular, $D^{p} f_{p+v}$ is v-Hölder continuous with constant

$$
\tilde{A}_{p, v}:= \begin{cases}(p-1) ! ! \prod_{i=1}^{p / 2}(v+2 i)+\prod_{i=1}^{p}(v+i), & \text { if } p \text { is even } \\ 2^{1-v} \prod_{i=1}^{p}(v+i), & \text { if } p \text { is odd }\end{cases}
$$

Proof First of all, observe that the constant $A_{p, v}$ is not smaller than the corresponding lower bound $C_{p, v}$ given by Theorem 6.1:

$$
C_{p, v} \leq A_{p, v}
$$

Indeed, for odd values of $p$, these constants coincide. When $p$ is even, (28) follows from the following trivial lower bound for the Hölder constant $H_{p, v}$ :

$$
H_{p, v} \geq g_{p, p+v}(1)-g_{p, p+v}(0)=\prod_{i=1}^{p}(v+i)-(p-1) ! ! \prod_{i=1}^{p / 2}(v+2 i)
$$

where the last equality is due to Proposition 4.2.

Second, observe that we only need to prove the first claim, since the other one follows directly from the first one and Theorem 5.2. 
Let $x_{1}, x_{2} \in \mathbb{E}$ and let $h \in \mathbb{E}$ be an arbitrary unit vector. In view of (1) and Theorem 3.1, we need to show that

$$
\left|\left\|x_{2}\right\|^{v} g_{p, p+v}\left(\tau_{2}\right)-\left\|x_{1}\right\|^{v} g_{p, p+v}\left(\tau_{1}\right)\right| \leq A_{p, v}\left\|x_{2}-x_{1}\right\|^{v}
$$

where $\tau_{1}:=\tau_{h}\left(x_{1}\right), \tau_{2}:=\tau_{h}\left(x_{2}\right)$.

Due to invariance of the above inequality to transformations of the form $\left(x_{1}, x_{2}\right) \mapsto$ $\left(x_{2}, x_{1}\right)$ and $\left(x_{1}, x_{2}\right) \mapsto\left(-x_{1},-x_{2}\right)$, we can assume in what follows that $\left\|x_{1}\right\| \leq\left\|x_{2}\right\|$ and $\tau_{2} \geq 0$. Furthermore, we can also assume that $x_{1} \neq 0$ (and hence $x_{2} \neq 0$ ), since otherwise the claim follows from Theorem 6.2.

There are now several cases to consider.

Case I Suppose $g_{p, p+v}\left(\tau_{1}\right)<0$. By Propositions 4.1 and 4.3, this happens only if $p$ is odd and $\tau_{1} \leq 0$. Then, $g_{p, p+v}\left(\tau_{1}\right)=-g_{p, p+v}\left(-\tau_{1}\right)$, and

$$
\left|\left\|x_{2}\right\|^{v} g_{p, p+v}\left(\tau_{2}\right)-\left\|x_{1}\right\|^{v} g_{p, p+v}\left(\tau_{1}\right)\right|=\left\|x_{2}\right\|^{v} g_{p, p+v}\left(\tau_{2}\right)+\left\|x_{1}\right\|^{v} g_{p, p+v}\left(-\tau_{1}\right),
$$

where we have removed the absolute value sign, because all terms in the right-hand side are nonnegative (see Proposition 4.3).

Since $p$ is odd, $g_{p, p+v}(0)=0$ (see Proposition 4.1). Therefore, by the definition of $H_{p, v}$, it follows that

$$
\begin{aligned}
g_{p, p+v}\left(-\tau_{1}\right) & =g_{p, p+v}\left(-\tau_{1}\right)-g_{p, p+v}(0) \leq H_{p, v}\left(-\tau_{1}^{v}\right), \\
g_{p, p+v}\left(\tau_{2}\right) & =g_{p, p+v}\left(\tau_{2}\right)-g_{p, p+v}(0) \leq H_{p, v} \tau_{2}^{v} .
\end{aligned}
$$

Combining this with the concavity of power function $t \mapsto t^{\nu}$, we obtain

$$
\begin{aligned}
\left\|x_{2}\right\|^{v} g_{p, p+v}\left(\tau_{2}\right)+\left\|x_{1}\right\|^{v} g_{p, p+v}\left(-\tau_{1}\right) & \leq H_{p, v}\left(\left(\left\|x_{2}\right\| \tau_{2}\right)^{v}+\left(-\left\|x_{1}\right\| \tau_{1}\right)^{v}\right) \\
& \leq 2^{1-v} H_{p, v}\left(\left\|x_{2}\right\| \tau_{2}-\left\|x_{1}\right\| \tau_{1}\right)^{v}
\end{aligned}
$$

Note that $2^{1-v} H_{p, v} \leq A_{p, v}$ by Theorem 5.2. Thus, it remains to show that $\left\|x_{2}\right\| \tau_{2}-$ $\left\|x_{1}\right\| \tau_{1} \leq\left\|x_{2}-x_{1}\right\|$. But this follows from the Cauchy-Schwartz inequality since $\left\|x_{2}\right\| \tau_{2}-\left\|x_{1}\right\| \tau_{1}=\left\langle B\left(x_{2}-x_{1}\right), h\right\rangle$ by the definition of $\tau_{1}, \tau_{2}$.

Case II Now suppose $g_{p, p+v}\left(\tau_{1}\right) \geq 0$ (while $\tau_{1}$ can have any sign). We prove (29) by proving separately two inequalities with the removed absolute value sign.

First, we show that

$$
\left\|x_{1}\right\|^{v} g_{p, p+v}\left(\tau_{1}\right)-\left\|x_{2}\right\|^{v} g_{p, p+v}\left(\tau_{2}\right) \leq A_{p, v}\left\|x_{2}-x_{1}\right\|^{v} .
$$

Let $x_{2}^{\prime}:=\frac{\left\|x_{1}\right\|}{\left\|x_{2}\right\|} x_{2}$ be the radial projection of $x_{2}$ onto the sphere with radius $r:=\left\|x_{1}\right\|$, centered at the origin. Note that

$$
\tau_{2}^{\prime}:=\tau_{h}\left(x_{2}^{\prime}\right)=\tau_{2}, \quad\left\|x_{2}^{\prime}\right\|=r \leq\left\|x_{2}\right\|, \quad\left\|x_{2}^{\prime}-x_{1}\right\| \leq\left\|x_{2}-x_{1}\right\| .
$$

The first two relations are evident. The last one follows from the fact that projections onto convex sets decrease distances and can be explicitly proved as follows. First, by 
the Cauchy-Schwartz inequality, we have $\left\langle B x_{1}, x_{2}\right\rangle \leq \rho\left\|x_{2}\right\|^{2}$, where $\rho:=\frac{\left\|x_{1}\right\|}{\left\|x_{2}\right\|} \leq 1$. Therefore, using the fact that $x_{2}^{\prime}=\rho x_{2}$, we obtain

$$
\begin{aligned}
\| x_{2} & -x_{1}\left\|^{2}-\right\| x_{2}^{\prime}-x_{1}\left\|^{2}=\right\| x_{2}\left\|^{2}-\right\| x_{2}^{\prime} \|^{2}-2\left\langle B x_{1}, x_{2}-x_{2}^{\prime}\right\rangle \\
& =\left(1-\rho^{2}\right)\left\|x_{2}\right\|^{2}-2(1-\rho)\left\langle B x_{1}, x_{2}\right\rangle \geq\left(1-\rho^{2}\right)\left\|x_{2}\right\|^{2}-2(1-\rho) \rho\left\|x_{2}\right\|^{2} \\
& =(1-\rho)^{2}\left\|x_{2}\right\|^{2} \geq 0 .
\end{aligned}
$$

Since $g_{p, p+v}\left(\tau_{2}\right) \geq 0$ (Proposition 4.3), from (31) it follows that

$$
\left\|x_{1}\right\|^{v} g_{p, p+v}\left(\tau_{1}\right)-\left\|x_{2}\right\|^{v} g_{p, p+v}\left(\tau_{2}\right) \leq r^{v}\left(g_{p, p+v}\left(\tau_{1}\right)-g_{p, p+v}\left(\tau_{2}^{\prime}\right)\right)
$$

At the same time, by Theorem 5.1,

$$
g_{p, p+v}\left(\tau_{1}\right)-g_{p, p+v}\left(\tau_{2}^{\prime}\right) \leq \tilde{H}_{p, v}\left|\tau_{2}^{\prime}-\tau_{1}\right|^{v}
$$

Hence,

$$
\left\|x_{1}\right\|^{v} g_{p, p+v}\left(\tau_{1}\right)-\left\|x_{2}\right\|^{v} g_{p, p+v}\left(\tau_{2}\right) \leq \tilde{H}_{p, v}\left(r\left|\tau_{2}^{\prime}-\tau_{1}\right|\right)^{v} .
$$

Note that

$$
r\left|\tau_{2}^{\prime}-\tau_{1}\right|=\left|\left\|x_{2}^{\prime}\right\| \tau_{2}^{\prime}-\left\|x_{1}\right\| \tau_{1}\right|=\left|\left\langle B\left(x_{2}^{\prime}-x_{1}\right), h\right\rangle\right| .
$$

Therefore, by Cauchy-Schwartz inequality and (31), we have

$$
r\left|\tau_{2}^{\prime}-\tau_{1}\right| \leq\left\|x_{2}^{\prime}-x_{1}\right\| \leq\left\|x_{2}-x_{1}\right\|
$$

Substituting this into (32), we obtain

$$
\left\|x_{1}\right\|^{v} g_{p, p+v}\left(\tau_{1}\right)-\left\|x_{2}\right\|^{\nu} g_{p, p+v}\left(\tau_{2}\right) \leq \tilde{H}_{p, v}\left\|x_{2}-x_{1}\right\|^{v} .
$$

This finishes the proof of (30), because $\tilde{H}_{p, v} \leq A_{p, v}$ by Theorem 5.2.

It remains to show the reverse inequality

$$
\left\|x_{2}\right\|^{v} g_{p, p+v}\left(\tau_{2}\right)-\left\|x_{1}\right\|^{v} g_{p, p+v}\left(\tau_{1}\right) \leq A_{p, v}\left\|x_{2}-x_{1}\right\|^{v} .
$$

For this, we consider two subcases.

Case II(a) Suppose $\tau_{1} \geq \tau_{2}$. Let $x_{1}^{\prime}:=\frac{\left\langle B x_{1}, x_{2}\right\rangle}{\left\|x_{2}\right\|^{2}} x_{2}$ be the projection of $x_{1}$ onto the line, connecting $x_{2}$ with the origin, and let $\tau_{1}^{\prime}:=\tau_{h}\left(x_{1}^{\prime}\right)$. Then,

$$
\left\|x_{1}^{\prime}\right\| \leq\left\|x_{1}\right\|, \quad\left\|x_{2}-x_{1}^{\prime}\right\| \leq\left\|x_{2}-x_{1}\right\|
$$

Furthermore,

$$
g_{p, p+v}\left(\tau_{1}^{\prime}\right) \leq g_{p, p+v}\left(\tau_{2}\right) .
$$


Indeed, if $\left\langle B x_{1}, x_{2}\right\rangle \geq 0$, then $\tau_{1}^{\prime}=\tau_{2}$ and $g_{p, p+v}\left(\tau_{1}^{\prime}\right)=g_{p, p+v}\left(\tau_{2}\right)$; otherwise, $\tau_{1}^{\prime}=-\tau_{2}$, and hence, $g_{p, p+v}\left(\tau_{1}^{\prime}\right)=(-1)^{p} g_{p, p+v}\left(\tau_{2}\right)$ (Proposition 4.1), which either coincides with $g_{p, p+v}\left(\tau_{2}\right)$ when $p$ is even, or becomes $-g_{p, p+v}\left(\tau_{2}\right) \leq 0$ when $p$ is odd (see Proposition 4.3).

Since $g_{p, p+v}\left(\tau_{2}\right) \leq g_{p, p+v}\left(\tau_{1}\right)$ (Proposition 4.4), it follows from (35) that $g_{p, p+v}\left(\tau_{1}^{\prime}\right) \leq g_{p, p+v}\left(\tau_{1}\right)$. Using also (34) and the fact that $g_{p, p+v}\left(\tau_{1}\right) \geq 0$, we obtain $\left\|x_{1}^{\prime}\right\|^{v} g_{p, p+v}\left(\tau_{1}^{\prime}\right) \leq\left\|x_{1}^{\prime}\right\|^{v} g_{p, p+v}\left(\tau_{1}\right) \leq\left\|x_{1}\right\|^{v} g_{p, p+v}\left(\tau_{1}\right)$. Thus,

$$
\left\|x_{2}\right\|^{v} g_{p, p+v}\left(\tau_{2}\right)-\left\|x_{1}\right\|^{v} g_{p, p+v}\left(\tau_{1}\right) \leq\left\|x_{2}\right\|^{v} g_{p, p+v}\left(\tau_{2}\right)-\left\|x_{1}^{\prime}\right\|^{v} g_{p, p+v}\left(\tau_{1}^{\prime}\right) \text {. }
$$

Note that in the right-hand side, we have the difference of the derivatives $D^{p} f_{p+v}\left(x_{2}\right)$ $[h]^{p}$ and $D^{p} f_{p+v}\left(x_{1}^{\prime}\right)[h]^{p}$, where the points $x_{1}^{\prime}$ and $x_{2}$ lie on a line, passing through the origin. Therefore, from Theorem 6.2, it follows that

$$
\left\|x_{2}\right\|^{v} g_{p, p+v}\left(\tau_{2}\right)-\left\|x_{1}\right\|^{v} g_{p, p+v}\left(\tau_{1}\right) \leq C_{p, v}\left\|x_{2}-x_{1}^{\prime}\right\|^{v}
$$

which proves (33), in view of (28) and (34).

Case II(b) Now suppose $\tau_{1} \leq \tau_{2}$. Denote by $\tilde{H}_{p, v}$ the constant of $v$-Hölder continuity of the polynomial $g_{p, p+v}$ on the interval $[-1,1]$. To prove $(33)$, it suffices to show that

$$
\begin{aligned}
& \left\|x_{2}\right\|^{v} g_{p, p+v}\left(\tau_{2}\right)-\left\|x_{1}\right\|^{v} g_{p, p+v}\left(\tau_{1}\right) \\
& \leq g_{p, p+v}(0)\left(\left\|x_{2}\right\|^{v}-\left\|x_{1}\right\|^{v}\right)+\tilde{H}_{p, v}\left(\left\|x_{2}\right\| \tau_{2}-\left\|x_{1}\right\| \tau_{1}\right)^{v},
\end{aligned}
$$

Indeed, recall that $\left\|x_{2}\right\|^{\nu}-\left\|x_{1}\right\|^{\nu} \leq\left\|x_{2}-x_{1}\right\|^{\nu}$. Also

$$
\left(\left\|x_{2}\right\| \tau_{2}-\left\|x_{1}\right\| \tau_{1}\right)^{\nu}=\left\langle B\left(x_{2}-x_{1}\right), h\right\rangle^{\nu} \leq\left\|x_{2}-x_{1}\right\|^{\nu}
$$

by the Cauchy-Schwartz inequality. Therefore, if (36) is true, then

$$
\left\|x_{2}\right\|^{v} g_{p, p+v}\left(\tau_{2}\right)-\left\|x_{1}\right\|^{v} g_{p, p+v}\left(\tau_{1}\right) \leq\left(g_{p, p+v}(0)+\tilde{H}_{p, v}\right)\left\|x_{2}-x_{1}\right\|^{v},
$$

where $g_{p, p+v}(0)+\tilde{H}_{p, v} \leq A_{p, v}$ in view of Proposition 4.2, Theorems 5.1 and 5.2. Thus, it remains to show (36), or, equivalently, that

$$
\begin{aligned}
& \left\|x_{2}\right\|^{v}\left(g_{p, p+v}\left(\tau_{2}\right)-g_{p, p+v}(0)\right) \\
& \quad \leq\left\|x_{1}\right\|^{v}\left(g_{p, p+v}\left(\tau_{1}\right)-g_{p, p+v}(0)\right)+\tilde{H}_{p, v}\left(\left\|x_{2}\right\| \tau_{2}-\left\|x_{1}\right\| \tau_{1}\right)^{v} .
\end{aligned}
$$

Denote $\rho:=\frac{\left\|x_{1}\right\|}{\left\|x_{2}\right\|} \in[0,1]$. We need to prove that

$$
g_{p, p+v}\left(\tau_{2}\right)-g_{p, p+v}(0) \leq \rho^{v}\left(g_{p, p+v}\left(\tau_{1}\right)-g_{p, p+v}(0)\right)+\tilde{H}_{p, v}\left(\tau_{2}-\rho \tau_{1}\right)^{v} .
$$


Note that the right-hand side of this inequality, as a function of $\rho \in[0,1]$, is concave (and well-defined, since $\tau_{1} \leq \tau_{2}$ ). Hence, to justify (37), we only need to prove the following two boundary cases:

$$
\begin{array}{ll}
\rho=0: & g_{p, p+v}\left(\tau_{2}\right)-g_{p, p+v}(0) \leq \tilde{H}_{p, v} \tau_{2}^{v} . \\
\rho=1: & g_{p, p+v}\left(\tau_{2}\right)-g_{p, p+v}\left(\tau_{1}\right) \leq \tilde{H}_{p, v}\left(\tau_{2}-\tau_{1}\right)^{v} .
\end{array}
$$

But both of them follow from the definition of $\tilde{H}_{p, v}$.

Comparing the result of Theorem 6.3 with the lower bound $C_{p, v}$, given by Theorem 6.1, we see that for odd values of $p$, the constant $\tilde{A}_{p, v}$ is optimal. Unfortunately, this is no longer true for even values of $p$. Nevertheless, the constant $\tilde{A}_{p, v}$ is still quite accurate. Indeed, since

$$
(p-1) ! !=\prod_{i=1}^{p / 2}(2 i-1) \leq \prod_{i=1}^{p / 2}(v+2 i-1)
$$

we have

$$
(p-1) ! ! \prod_{i=1}^{p / 2}(v+2 i) \leq \prod_{i=1}^{p}(v+i)
$$

Thus, the constant $\tilde{A}_{p, v}$ is at most two times suboptimal: $\tilde{A}_{p, v} \leq 2 C_{p, v}$.

One may think that the reason, why we obtained a suboptimal bound for even values of $p$, is related to the fact that we had used a suboptimal value for the Hölder constant $H_{p, v}$ of the polynomial $g_{p, p+v}$ (see the corresponding discussion at the end of Sect. 5). However, this is not the actual reason. Indeed, let us look what happens when we use the optimal value for $H_{p, v}$ in the particular case $p=2$. Recall that the optimal constant in this case is

$$
H_{2, v}=v(v+2) \frac{2^{2-v}(1-v)^{1-v}}{(2-v)^{2-v}} .
$$

Substituting this expression into (27), we obtain an improved estimate

$$
A_{2, v}=v+2+H_{2, v}=(v+2)\left(v+1+v \frac{2^{2-v}(1-v)^{1-v}}{(2-v)^{2-v}}\right) \text {. }
$$

However, this new estimate is still different from the lower bound

$$
C_{2, v}=(v+1)(v+2)
$$

At the same time, for small values of $v$, the difference between $A_{p, v}$ and $C_{p, v}$ is almost negligible. 


\section{Lipschitz Constants of Derivatives of Powers of Euclidean Norm}

For even values of $p$, our estimate $A_{p, v}$ of the Hölder constant of $D^{p} f_{p+v}$ was suboptimal. It turns out that in the special case when $v=1$, it is actually very simple to eliminate this drawback and obtain an optimal constant for all values of $p$. This case corresponds to Lipschitz continuity.

Theorem 7.1 For any integer $p \geq 0$, the derivative $D^{p} f_{p+1}$ is Lipschitz continuous with constant

$$
C_{p, 1}=(p+1) !
$$

where $n$ ! for a nonnegative integer $n$ denotes the factorial of $n$.

Proof It suffices to prove that $\left|D^{p+1} f_{p+1}(x)[h]^{p+1}\right| \leq(p+1)$ ! for all $x \in \mathbb{E}$ and all unit $h \in \mathbb{E}$. By Theorem 3.1, we have $D^{p+1} f_{p+1}(x)[h]^{p+1}=g_{p+1, p+1}\left(\tau_{h}(x)\right)$. Since $\left|\tau_{h}(x)\right| \leq 1$, we obtain $\left|D^{p+1} f_{p+1}(x)[h]^{p+1}\right| \leq \max _{[-1,1]}\left|g_{p+1, p+1}\right|$. The claim now follows from Proposition 4.5.

\section{Conclusions}

In this work, we have proved that derivatives of powers of Euclidean norm are Hölder continuous and have obtained explicit expressions for the corresponding Hölder constants. We have shown that our constants are optimal for odd derivatives and at most two times suboptimal for the even ones. In the particular case of integer powers, when the Hölder condition corresponds to the Lipschitz condition, we have managed to improve our result and obtained optimal constants in all cases. We believe that in general, it should be possible to obtain optimal constants for even derivatives as well. However, this seems to be a difficult problem.

Acknowledgements The presented results were supported by ERC Advanced Grant 788368. We are grateful to the anonymous referees for their attentive reading and valuable comments.

Open Access This article is licensed under a Creative Commons Attribution 4.0 International License, which permits use, sharing, adaptation, distribution and reproduction in any medium or format, as long as you give appropriate credit to the original author(s) and the source, provide a link to the Creative Commons licence, and indicate if changes were made. The images or other third party material in this article are included in the article's Creative Commons licence, unless indicated otherwise in a credit line to the material. If material is not included in the article's Creative Commons licence and your intended use is not permitted by statutory regulation or exceeds the permitted use, you will need to obtain permission directly from the copyright holder. To view a copy of this licence, visit http://creativecommons.org/licenses/by/4.0/.

\section{References}

1. Nesterov, Y., Polyak, B.T.: Cubic regularization of Newton method and its global performance. Math. Program. 108(1), 177-205 (2006)

2. Nesterov, Y.: Accelerating the cubic regularization of Newton's method on convex problems. Math. Program. 112(1), 159-181 (2008) 
3. Cartis, C., Gould, N.I., Toint, P.L.: Adaptive cubic regularisation methods for unconstrained optimization. Part I: motivation, convergence and numerical results. Math. Program. 127(2), 245-295 (2011)

4. Cartis, C., Gould, N.I., Toint, P.L.: Adaptive cubic regularisation methods for unconstrained optimization. Part II: worst-case function-and derivative-evaluation complexity. Math. Program. 130(2), 295-319 (2011)

5. Carmon, Y., Duchi, J.C.: Gradient descent efficiently finds the cubic-regularized non-convex Newton step. arXiv preprint arXiv:1612.00547 (2016)

6. Kohler, J.M., Lucchi, A.: Sub-sampled cubic regularization for non-convex optimization. In: Proceedings of the 34th International Conference on Machine Learning, vol. 70, pp. 1895-1904. JMLR (2017)

7. Cartis, C., Scheinberg, K.: Global convergence rate analysis of unconstrained optimization methods based on probabilistic models. Math. Program. 169(2), 337-375 (2018)

8. Doikov, N., Richtarik, P., et al.: Randomized block cubic Newton method. In: International Conference on Machine Learning, pp. 1289-1297 (2018)

9. Schnabel, R.B., Chow, T.T.: Tensor methods for unconstrained optimization using second derivatives. SIAM J. Optim. 1(3), 293-315 (1991)

10. Baes, M.: Estimate sequence methods: extensions and approximations. Optimization Online (2009)

11. Cartis, C., Gould, N.I., Toint, P.L.: Improved second-order evaluation complexity for unconstrained nonlinear optimization using high-order regularized models. arXiv preprint arXiv:1708.04044 (2017)

12. Gasnikov, A., Dvurechensky, P., Gorbunov, E., Vorontsova, E., Selikhanovych, D., Uribe, C.A., Jiang, B., Wang, H., Zhang, S., Bubeck, S., et al.: Near optimal methods for minimizing convex functions with Lipschitz $p$-th derivatives. In: Conference on Learning Theory, pp. 1392-1393 (2019)

13. Nesterov, Y.: Implementable tensor methods in unconstrained convex optimization. Technical report, CORE discussion paper, Université Catholique de Louvain, Belgium (2015)

14. Grapiglia, G.N., Nesterov, Y.: Regularized Newton methods for minimizing functions with Hölder continuous Hessians. SIAM J. Optim. 27(1), 478-506 (2017)

15. Grapiglia, G.N., Nesterov, Y.: Tensor methods for minimizing functions with Hölder continuous higherorder derivatives. arXiv preprint arXiv:1904.12559 (2019)

16. Vladimirov, A., Nesterov, Y.E., Chekanov, Y.N.: On uniformly convex functionals. Vestnik Moskov. Univ. Ser. XV Vychisl. Mat. Kibernet 3, 12-23 (1978)

17. Doikov, N., Nesterov, Y.: Minimizing uniformly convex functions by cubic regularization of Newton method. arXiv preprint arXiv:1905.02671 (2019)

18. Nesterov, Y.: Universal gradient methods for convex optimization problems. Math. Program. 152(1-2), 381-404 (2015)

19. Nesterov, Y., Nemirovskii, A.: Interior-Point Polynomial Algorithms in Convex Programming, vol. 13. SIAM, Philadelphia (1994)

Publisher's Note Springer Nature remains neutral with regard to jurisdictional claims in published maps and institutional affiliations. 